\title{
Perturbative unitarity bounds on di-boson scalar resonances
}

\author{
Luca Di Luzio, a, Jernej F. Kamenik ${ }^{2,3, b}$, and Marco Nardecchia ${ }^{4, c}$ \\ ${ }_{1}^{1}$ Institute for Particle Physics Phenomenology, Department of Physics, Durham University, DH1 3LE, UK \\ ${ }^{2}$ Jožef Stefan Institute, Jamova 39, 1000 Ljubljana, Slovenia \\ ${ }^{3}$ Faculty of Mathematics and Physics, University of Ljubljana, Jadranska 19, 1000 Ljubljana, Slovenia \\ ${ }^{4}$ CERN, Theoretical Physics Department, Geneva, Switzerland
}

\begin{abstract}
We consider the constraints implied by partial wave unitarity on new physics in the form of di-boson resonances at LHC. We derive the scale where the effective description in terms of the SM supplemented by a single scalar resonance is expected to break down depending on the resonance width and signal cross-section.
\end{abstract}

\section{Introduction}

Perturbative unitarity is a powerful theoretical tool for inferring the range of validity of a given effective field theory (EFT), with notable examples of applications both in the physics of strong and electroweak interactions. Perhaps most famously, constraints imposed by perturbative unitarity in $W W$ scattering have been used in the past to infer an upper bound on the Higgs boson mass or, alternatively, on the scale where the standard model (SM) description of weak interactions would need to be completed in the ultraviolet (UV) in terms of some new strongly coupled dynamics [1].

The recently rekindled interest in new physics (NP) in the form of (possibly broad) di-photon resonances [2,3] at the LHC motivated us to reconsider the implications of perturbative unitarity for EFT interpretations of resonances decaying to di-boson final states. In particular, focusing on promptly produced scalar SM singlets decaying to two SM gauge bosons we aim to address the following question: at which maximal energies do we expect the effective description in terms of the SM supplemented by a single scalar to break down? Assuming that a scalar resonance is within the reach of the present LHC run, can a next generation hadron collider potentially probe the on-shell effects of new degrees of freedom responsible for the restoration of unitarity?

After a brief recap of partial wave unitarity arguments in Sect. 2, we apply them in Sect. 3 to the EFT case where a di-boson resonance is the only new degree of freedom beyond the SM. The scale of unitarity violation is hence interpreted as an upper bound on the mass scale of new degrees of freedom UV completing the effective low-energy description and regularizing (unitarizing) the amplitudes' growth. The present contribution is largely based on Ref. [4], to which the reader is referred for further details.

\footnotetext{
a e-mail: luca.di-luzio@durham.ac.uk

be-mail: jernej.kamenik@cern.ch

ce-mail: marco.nardecchia@cern.ch
} 


\section{Partial wave unitarity}

Let us denote by $\mathcal{T}_{f i}(\sqrt{s}, \cos \theta)$ the matrix element of a $2 \rightarrow 2$ scattering amplitude in momentum space, defined via

$$
(2 \pi)^{4} \delta^{(4)}\left(P_{i}-P_{f}\right) \mathcal{T}_{f i}(\sqrt{s}, \cos \theta)=\langle f|T| i\rangle,
$$

where $T$ is the interacting part of the $S$-matrix, $S=1+i T$. The dependence of the scattering amplitude on $\cos \theta$ is eliminated by projecting it onto partial waves of total angular momentum $J$

$$
a_{f i}^{J}=\frac{\beta_{f}^{1 / 4}\left(s, m_{f 1}^{2}, m_{f 2}^{2}\right) \beta_{i}^{1 / 4}\left(s, m_{i 1}^{2}, m_{i 2}^{2}\right)}{32 \pi s} \int_{-1}^{1} d(\cos \theta) d_{\mu_{i} \mu_{f}}^{J}(\theta) \mathcal{T}_{f i}(\sqrt{s}, \cos \theta),
$$

where $d_{\mu_{i} \mu_{f}}^{J}$ is the $J$-th Wigner $d$-function appearing in the Jacob-Wick expansion [5], while $\mu_{i}=$ $\lambda_{i 1}-\lambda_{i 2}$ and $\mu_{f}=\lambda_{f 1}-\lambda_{f 2}$ are defined in terms of the helicities of the initial $\left(\lambda_{i 1}, \lambda_{i 2}\right)$ and final $\left(\lambda_{f 1}, \lambda_{f 2}\right)$ states. The function $\beta(x, y, z)=x^{2}+y^{2}+z^{2}-2 x y-2 y z-2 z x$ is a kinematical factor related to the momentum (to the fourth power) of a given particle in the center of mass frame. The right hand side of Eq. (2) must be further multiplied by a $\frac{1}{\sqrt{2}}$ factor for any identical pair of particles either in the initial or final state.

When restricted to a same-helicity state (zero total spin), the Wigner $d$-functions reduce to the Legendre polynomials, i.e. $d_{00}^{J}=P_{J}$. In practice, we will only focus on $J=0\left(d_{00}^{0}=P_{0}=1\right)$, since higher partial waves typically give smaller amplitudes, unless $J=0$ amplitudes are suppressed or vanish for symmetry reasons. Hence, the quantity we are interested in is

$$
a_{f i}^{0}=\frac{\beta_{f}^{1 / 4}\left(s, m_{f 1}^{2}, m_{f 2}^{2}\right) \beta_{i}^{1 / 4}\left(s, m_{i 1}^{2}, m_{i 2}^{2}\right)}{32 \pi s} \int_{-1}^{1} d(\cos \theta) \mathcal{T}_{f i}(\sqrt{s}, \cos \theta) .
$$

In the high-energy limit, $\sqrt{s} \rightarrow \infty$, one has $\beta_{f}^{1 / 4} \beta_{i}^{1 / 4} / s \rightarrow 1$. The unitarity condition on the $S$-matrix, $S S^{\dagger}=1$, gives

$$
\frac{1}{2 i}\left(a_{f i}^{J}-a_{i f}^{J *}\right) \geq \sum_{h} a_{h f}^{J *} a_{h i}^{J}
$$

where the sum over $h$ is restricted to 2-particle states, which slightly underestimates the left hand side. For $i=f$ Eq. (4) reduces to

$$
\operatorname{Im} a_{i i}^{J} \geq\left|a_{i i}^{J}\right|^{2} .
$$

Hence, $a_{i i}^{J}$ must lie inside the circle in the Argand plane defined by

$$
\left(\operatorname{Re} a_{i i}^{J}\right)^{2}+\left(\operatorname{Im} a_{i i}^{J}-\frac{1}{2}\right)^{2} \leq \frac{1}{4}
$$

which implies

$$
\left|\operatorname{Im} a_{i i}^{J}\right| \leq 1 \quad \text { and } \quad\left|\operatorname{Re} a_{i i}^{J}\right| \leq \frac{1}{2} .
$$

Under the assumption that the tree-level amplitude is real, Eq. (7) suggests the following perturbativity criterium

$$
\left|\operatorname{Re}\left(a_{i i}^{J}\right)^{\text {Born }}\right| \leq \frac{1}{2} .
$$

In fact, a Born value of $\operatorname{Re} a_{i i}^{J}=\frac{1}{2}$ and $\operatorname{Im} a_{i i}^{J}=0$ needs at least a correction of $40 \%$ in order to restore unitarity, thus signalling the breakdown of the $(E / \Lambda)$ power expansion of the EFT, where $E$ is the typical energy involved in a process and $\Lambda$ is the EFT cut-off scale. 


\section{Effective field theory of a scalar resonance}

We consider the EFT of a gauge singlet spin-0 resonance, $S$ with mass $M_{S}$, coupled to the SM fields. Assuming CP invariance, we choose $S$ to transform as a scalar. ${ }^{1}$ Apart from renormalizable terms coupling $S$ to the Higgs in the scalar potential, the $d=5$ Lagrangian reads

$$
\begin{aligned}
\mathcal{L}_{\text {int. }}^{(5)}= & -\frac{g_{3}^{2}}{2 \Lambda_{g}} S G_{\mu \nu}^{2}-\frac{g_{2}^{2}}{2 \Lambda_{W}} S W_{\mu \nu}^{2}-\frac{g_{1}^{2}}{2 \Lambda_{B}} S B_{\mu \nu}^{2}-\frac{1}{\Lambda_{H}} S\left(D_{\mu} H\right)^{\dagger} D^{\mu} H-\frac{1}{\Lambda_{H}^{\prime}} S\left(H^{\dagger} H\right)^{2} \\
& -\frac{1}{\Lambda_{d}} S \bar{Q}_{L} d_{R} H-\frac{1}{\Lambda_{u}} S \bar{Q}_{L} u_{R} H^{c}-\frac{1}{\Lambda_{e}} S \bar{L}_{L} e_{R} H+\text { h.c. },
\end{aligned}
$$

where we have suppressed flavor indices. This parametrization makes it clear that the leading interactions of a scalar singlet with the SM fields, directly relevant for di-boson resonances at the LHC, are all due to non-renormalizable $d=5$ operators. Their effects are thus expected to be enhanced at high energies eventually leading to the breakdown of perturbative unitarity. In order to quantify this simple observation in the following subsections we evaluate the relevant scattering amplitudes involving SM gauge bosons, Higgs and quarks at the respective leading orders in perturbation theory. Moreover, since we are interested in studying $2 \rightarrow 2$ scattering processes at energies $\sqrt{s} \gg M_{S} \gg v \simeq 246 \mathrm{GeV}$, we can safely set all the massive parameters (including $M_{S}$ ) to zero and work within the unbroken SM theory. This also implies that we can neglect any $h-S$ mixing effects and set the masses of the final state SM particles to zero. We distinguish between two classes of tree-level processes characterized by a different energy scaling of the amplitude: scalar mediated scatterings and $d=5$ contact interactions.

\subsection{Scalar mediated boson scattering}

Let us start, as an example, by considering the $\gamma \gamma \rightarrow \gamma \gamma$ scattering amplitude due to the effective operator

$$
-\frac{e^{2}}{2 \Lambda_{\gamma}} S F_{\mu \nu}^{2}
$$

In the $(++,--)$ helicity basis we find [4]

$$
\mathcal{T}=-\frac{e^{4}}{\Lambda_{\gamma}^{2}}\left(\begin{array}{cc}
\frac{s^{2}}{s-M_{S}^{2}} & \frac{s^{2}}{s-M_{S}^{2}}+\frac{t^{2}}{t-M_{S}^{2}}+\frac{u^{2}}{u-M_{S}^{2}} \\
\frac{s^{2}}{s-M_{S}^{2}}+\frac{t^{2}}{t-M_{S}^{2}}+\frac{u^{2}}{u-M_{S}^{2}} & \frac{s^{2}}{s-M_{S}^{2}}
\end{array}\right) \sqrt{s} \gg M_{S}-\frac{e^{4} s}{\Lambda_{\gamma}^{2}}\left(\begin{array}{ll}
1 & 0 \\
0 & 1
\end{array}\right),
$$

where in the last step we took the high-energy limit, and only the $s$-channel survives at high energies.

The projection on the $J=0$ partial waves is obtained by applying Eq. (3) and by multiplying by a $1 / 2$ factor which takes into account the presence of identical particles both in the initial and final states. In the high-energy limit we get

$$
a^{0} \simeq-\frac{e^{4} s}{32 \pi \Lambda_{\gamma}^{2}}\left(\begin{array}{ll}
1 & 0 \\
0 & 1
\end{array}\right)
$$

which, confronted with Eq. (8), leads to the tree-level unitarity bound

$$
\sqrt{s} \lesssim \sqrt{16 \pi} \frac{\Lambda_{\gamma}}{e^{2}} .
$$

\footnotetext{
${ }^{1}$ The pseudo-scalar case leads to analogous conclusions as far as unitarity bounds are concerned, hence in the following we will not consider it separately.
} 
As a matter of fact, the bound above can be made stronger if one considers the full $V V \rightarrow V^{\prime} V^{\prime}$ scattering matrix, where $V$ and $V^{\prime}$ are any of the $8+3+1$ (transversely polarized) SM gauge bosons of the effective Lagrangian in Eq. (9). In such a case, the previous calculation is readily generalized in the high-energy limit where only the $s$-channel survives. To this end, we note that a scattering amplitude in the $s$-channel can be written as

$$
m_{i j}=\frac{a_{i} a_{j}}{s-M_{S}^{2}},
$$

where $a_{i}$ and $a_{j}$ are obtained by cutting any $i \rightarrow j$ diagram in two parts along the $s$-channel propagator. The matrix in Eq. (14) has rank 1 and its non-zero eigenvalue is given by the trace. Hence, denoting by $\tilde{a}^{0}$ the eigenvalue of the $V V \rightarrow V^{\prime} V^{\prime}$ scattering matrix, in the high-energy limit we get

$$
\tilde{a}^{0} \simeq-\frac{s}{32 \pi}\left(\frac{8 g_{3}^{4}}{\Lambda_{g}^{2}}+\frac{3 g_{2}^{4}}{\Lambda_{W}^{2}}+\frac{g_{1}^{4}}{\Lambda_{B}^{2}}\right)
$$

Correspondingly, the tree-level unitarity bound is given by

$$
\frac{s}{32 \pi}\left(8 \frac{g_{s}^{4}}{\Lambda_{g}^{2}}+3 \frac{g_{2}^{4}}{\Lambda_{W}^{2}}+\frac{g_{1}^{4}}{\Lambda_{B}^{2}}\right) \lesssim \frac{1}{2} .
$$

We remark that in deriving these bounds we consider only the transverse polarizations of the $W$ and $Z$ gauge bosons. Generally, scattering amplitudes involving longitudinally polarized massive vector bosons can grow as positive powers of $E / m_{W, Z}$ implying apparently stronger dependence on $s$. However, as it can be easily verified (through an explicit calculation of the processes at hand or more generally via a clever gauge choice [6]), the scattering amplitudes involving longitudinally polarized states sourced by the gauge field strengths in Eq. (9) are suppressed by powers of $m_{W, Z} / E$ and thus do not lead to relevant unitarity constraints at high $s$.

\subsection{Fermion-scalar contact interactions}

Next we consider the contact interaction

$$
-\frac{1}{\Lambda_{d}} S \bar{Q}_{L} d_{R} H=\left[-\frac{1}{\Lambda_{d}} \delta_{a}^{b} \delta_{i}^{j}\right] S\left(\bar{Q}_{L}\right)^{a i}\left(d_{R}\right)_{b} H_{j},
$$

where we have explicitly factored out the color and $S U(2)_{L}$ group structure. In this case the leading scattering process is $\bar{Q} d \rightarrow S H$. By explicitly writing the polarization and gauge indices in the amplitude, one finds

$$
\mathcal{T}=-\frac{\delta_{a}^{b} \delta_{i}^{j}}{2 \Lambda_{d}} \bar{v}^{s}(k)\left(1+\gamma_{5}\right) u^{r}(p) .
$$

Only the ++ and -- polarizations survive. By explicit evaluation we get [4]

$$
\begin{aligned}
& \mathcal{T}_{++}=\frac{\delta_{a}^{b} \delta_{i}^{j}}{\Lambda_{d}}\left(E+p^{3}\right) \stackrel{\sqrt{s} \gg M_{S}}{\simeq} \delta_{a}^{b} \delta_{i}^{j} \frac{\sqrt{s}}{\Lambda_{d}}, \\
& \mathcal{T}_{--}=\frac{\delta_{a}^{b} \delta_{i}^{j}}{\Lambda_{d}}\left(E-p^{3}\right) \stackrel{\sqrt{s} \gg M_{S}}{\simeq} 0 .
\end{aligned}
$$


At high energies the $J=0$ partial wave is obtained by considering the color singlet channel for a state in the linear combination $\frac{1}{\sqrt{2}}(|\bar{Q} d\rangle+|S H\rangle)$, which gives

$$
a^{0} \simeq \frac{1}{16 \pi} \frac{\sqrt{s}}{\Lambda_{d}} .
$$

Correspondingly, the tree-level unitarity bound reads

$$
\sqrt{s} \lesssim 8 \pi \Lambda_{d}
$$

Similarly, from the other two contact interactions in the last row of Eq. (9) we get $\sqrt{s} \lesssim 8 \pi \Lambda_{u, e}$.

\subsection{Unitarity bounds}

As an exemplification we consider a scalar resonance $S$ with mass $M_{S}$ and total width $\Gamma_{S}$ appearing in a di-photon final state at the LHC. ${ }^{2}$ Expanding the effective Lagrangian in Eq. (9) around the broken electroweak (EW) vacuum, the part relevant for $S$ production at the LHC is

$$
\mathcal{L}_{\text {int. }}^{(5)} \supset-\frac{g_{3}^{2}}{2 \Lambda_{g}} S G_{\mu \nu}^{2}-\frac{e^{2}}{2 \Lambda_{\gamma}} S F_{\mu \nu}^{2}-\sum_{q} y_{q S} S \bar{q} q,
$$

whose operators give rise to the decay widths

$$
\begin{aligned}
& \Gamma_{\gamma \gamma} \equiv \Gamma(S \rightarrow \gamma \gamma)=\pi \alpha_{\mathrm{EM}}^{2} \frac{M_{S}^{3}}{\Lambda_{\gamma}^{2}} \\
& \Gamma_{g g} \equiv \Gamma(S \rightarrow g g)=8 \pi \alpha_{s}^{2} \frac{M_{S}^{3}}{\Lambda_{g}^{2}}, \\
& \Gamma_{q \bar{q}} \equiv \Gamma(S \rightarrow q \bar{q})=\frac{3}{8 \pi} y_{q S}^{2} M_{S}\left(1-\frac{4 m_{q}^{2}}{M_{S}^{2}}\right)^{3 / 2} .
\end{aligned}
$$

The matching between the operators in Eq. (23) and Eq. (9) then yields

$$
\frac{1}{\Lambda_{\gamma}}=\frac{1}{\Lambda_{B}}+\frac{1}{\Lambda_{W}}, \quad y_{q S}=\frac{v}{\sqrt{2} \Lambda_{q}} .
$$

In the narrow width approximation the prompt $S$ production at the LHC can also be fully parametrized in terms of the relevant decay widths

$$
\sigma(p p \rightarrow S)=\frac{1}{M_{S} s}\left[\sum_{\mathcal{P}} C_{\mathcal{P} \overline{\mathcal{P}}} \Gamma_{\mathcal{P} \overline{\mathcal{P}}}\right],
$$

where $\sqrt{s}$ is the LHC $p p$ collision energy and $C_{\bar{\rho} \overline{\mathcal{p}}}$ parametrize the relevant parton luminosities.

For illustration purposes in the following we consider in turn either $g g$ and $\gamma \gamma$ induced processes or alternatively $b \bar{b}$ and $\gamma \gamma$ rates at a benchmark mass of $M_{S}=750 \mathrm{GeV}$. The remaining possibilities

\footnotetext{
${ }^{2}$ Analogous analysis can be performed also for other EW gauge boson final states with the slight complication of disentangling the transverse and longitudinal gauge boson polarizations, as they are sourced by different terms in the EFT Lagrangian $\left(\Lambda_{B, W}\right.$ and $\Lambda_{H}$, respectively).
} 
lie in between these two limiting cases considering the values of relevant parton luminosities (their values at $\sqrt{s}=8 \mathrm{TeV}$ and $13 \mathrm{TeV}$ LHC can be found e.g. in [7]). In the former case given a $13 \mathrm{TeV}$ cross-section $\sigma_{\gamma \gamma} \equiv \sigma(p p \rightarrow S) \mathcal{B}_{\gamma \gamma}$ one obtains the relation

$$
\frac{\Gamma_{\gamma \gamma}}{M_{S}} \frac{\Gamma_{g g}}{M_{S}} \simeq 1.4 \times 10^{-7} \frac{\sigma_{\gamma \gamma}}{\mathrm{fb}} \frac{\Gamma_{S}}{M_{S}}
$$

while for the latter we obtain

$$
\frac{\Gamma_{\gamma \gamma}}{M_{S}} \frac{\Gamma_{b \bar{b}}}{M_{S}} \simeq 2.3 \times 10^{-5} \frac{\sigma_{\gamma \gamma}}{\mathrm{fb}} \frac{\Gamma_{S}}{M_{S}} .
$$

These relations define the phenomenological benchmarks for the resonance partial widths into gauge boson and quark final states, to be subjected to constraints from perturbative unitarity.

To make contact with the EFT unitarity discussion of the preceding subsections we use Eqs. (24)(25) and trade $\Lambda_{g}, \Lambda_{W}$ and $\Lambda_{B}$ for $\Gamma_{g g}, \Gamma_{\gamma \gamma}$ and the ratio $r \equiv \Lambda_{B} / \Lambda_{W}$. In particular, we get

$$
\frac{1}{\Lambda_{g}^{2}}=\frac{\Gamma_{g g}}{8 \pi \alpha_{s}^{2} M_{S}^{3}}, \quad \frac{1}{\Lambda_{W}^{2}}=\frac{\Gamma_{\gamma \gamma}}{\pi \alpha_{\mathrm{EM}}^{2} M_{S}^{3}}\left(\frac{r}{1+r}\right)^{2}, \quad \frac{1}{\Lambda_{B}^{2}}=\frac{\Gamma_{\gamma \gamma}}{\pi \alpha_{\mathrm{EM}}^{2} M_{S}^{3}}\left(\frac{1}{1+r}\right)^{2},
$$

which inserted back into Eq. (16) yield

$$
\sqrt{s} \lesssim M_{S}\left(\frac{\Gamma_{g g}}{M_{S}}+f(r) \frac{\Gamma_{\gamma \gamma}}{M_{S}}\right)^{-1 / 2},
$$

with

$$
f(r)=\frac{3 r^{2} s_{W}^{-4}+c_{W}^{-4}}{(1+r)^{2}} .
$$

In the phenomenologically interesting range $r \in[-0.3,15][7,8]$, the function $f(r)$ has the minimal value 1.6 for $r=0.030$ and the maximal one 50 at $r=15$. Hence, we can set the following unitarity bounds

$$
\begin{aligned}
& \sqrt{s} \lesssim 32 M_{S}\left(\frac{\Gamma_{g g} / M_{S}}{10^{-3}}\right)^{-1 / 2}, \\
& \sqrt{s} \lesssim(15 \div 80) M_{S}\left(\frac{\Gamma_{\gamma \gamma} / M_{S}}{10^{-4}}\right)^{-1 / 2},
\end{aligned}
$$

where the values 15 and 80 in the last equation correspond respectively to $r=15$ and 0.030 .

In the case of quark scattering, we use Eq. (26) and Eq. (27). Thus the bound in Eq. (22) translates into

$$
\sqrt{s} \lesssim 2 \sqrt{3 \pi} v\left(\frac{\Gamma_{q \bar{q}}}{M_{S}}\right)^{-1 / 2} \simeq 4.8 \mathrm{TeV}\left(\frac{\Gamma_{q \bar{q}} / M_{S}}{0.1}\right)^{-1 / 2},
$$

where on the r.h.s. we have normalized the partial width in $q \bar{q}$ to a broad resonance scenario.

In Fig. 1 we display the scale of unitarity violation $\Lambda_{U}[\mathrm{TeV}]$ in the $\mathcal{B}_{\gamma \gamma}$ vs. $\sigma_{\gamma \gamma}$ plane, for either $g g$ or $b \bar{b}$ production and assuming either a broad or narrow resonance. In particular, for $g g$ production we have

$$
\Lambda_{U}=M_{S}\left[\frac{\Gamma_{g g}}{M_{S}}+f(r) \frac{\Gamma_{\gamma \gamma}}{M_{S}}\right]^{-1 / 2}
$$


while for $b \bar{b}$ production

$$
\Lambda_{U}=\min \left\{2 \sqrt{3 \pi} v\left(\frac{\Gamma_{b \bar{b}}}{M_{S}}\right)^{-1 / 2}, M_{S}\left[f(r) \frac{\Gamma_{\gamma \gamma}}{M_{S}}\right]^{-1 / 2}\right\} .
$$

As reference values we take $M_{S}=750 \mathrm{GeV}$ and $f(r)=30$ The horizontal lines from top to bottom indicate a cross-section signal of $6,0.6$ and $0.2 \mathrm{fb}$, assuming the same significance of the signal over the three integrated luminosities $\int \mathcal{L}=3.2,300$ and $3000 \mathrm{fb}^{-1}$. The red curve denotes instead the reference value $\Lambda=20 \mathrm{TeV}$, corresponding to the typical squark-gluino reach of a futuristic $100 \mathrm{TeV}$ collider [9], which applies in the case of coloured new physics generating the effective operators. Hence, if a signal is observed above the red curve it basically means that a $100 \mathrm{TeV}$ collider could potentially probe the physics responsible for the restoration of unitarity. We observe that such lowscale violation of unitarity are more readily obtained in the large width scenario and that for any given $\sigma_{\gamma \gamma}$ and $\mathcal{B}_{\gamma \gamma}$, unitarity violation sets in earlier for $b \bar{b}$ induced production, compared to gluon fusion processes, due to much smaller PDFs.

\section{Conclusions}

In the case of a TeV-scale scalar di-boson resonance observable at the LHC we have, under some very basic and natural assumptions on the structure of the EFT (mainly that $S$ is a spin-0 SM gauge singlet and that the $d=5$ operators in Eq. (9) are the most relevant ones for the decay of $S$ ), demonstrated a potential violation of tree-level unitarity in the scattering of SM fields at energy scales of few tens of $\mathrm{TeV}$. This scale can be interpreted as an upper bound on the mass of new degrees of freedom responsible for the unitarization of the scattering amplitudes.

We conclude that in the event of an experimental observation of a scalar di-boson resonance at the LHC, while our estimates cannot provide a guarantee to see on-shell effects of additional new degrees of freedom at the LHC, they would immediately imply the existence of additional phenomena within the energy reach of the next generation 50-100 TeV hadron colliders, thus making a strong physics case for their construction.

\section{Acknowledgments}

L.D.L. wishes to thank the organizers of the ICNFP 2016 for providing a wonderful atmosphere on the Crete island. J.F.K. acknowledges the financial support from the Slovenian Research Agency (research core funding No. P1-0035).

\section{References}

[1] B.W. Lee, C. Quigg, H.B. Thacker, Phys. Rev. Lett. 38, 883 (1977)

[2] M. Aaboud et al. (ATLAS), JHEP 09, 001 (2016), 1606.03833

[3] V. Khachatryan et al. (CMS), Phys. Rev. Lett. 117, 051802 (2016), 1606.04093

[4] L. Di Luzio, J.F. Kamenik, M. Nardecchia (2016), 1604.05746

[5] M. Jacob, G.C. Wick, Annals Phys. 7, 404 (1959), [Annals Phys.281,774(2000)]

[6] A. Wulzer, Nucl. Phys. B885, 97 (2014), 1309.6055

[7] R. Franceschini, G.F. Giudice, J.F. Kamenik, M. McCullough, A. Pomarol, R. Rattazzi, M. Redi, F. Riva, A. Strumia, R. Torre, JHEP 03, 144 (2016), 1512 . 04933

[8] J.F. Kamenik, B.R. Safdi, Y. Soreq, J. Zupan, JHEP 07, 042 (2016), 1603.06566

[9] T. Golling et al. (2016), 1606.00947 

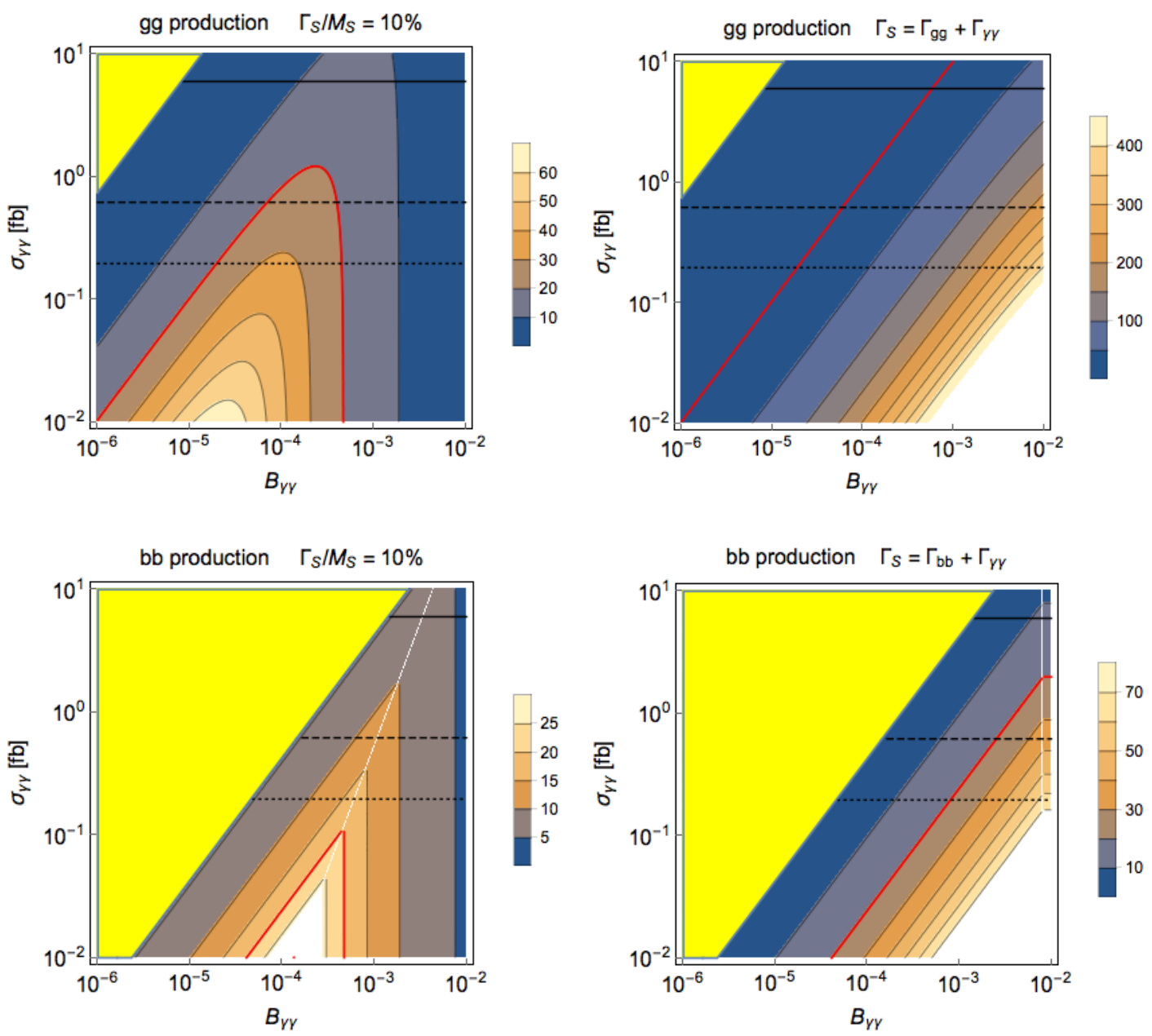

Figure 1: Scale of unitarity violation $\Lambda_{U}$ in $\mathrm{TeV}$ in the $\left(\mathcal{B}_{\gamma \gamma}, \sigma_{\gamma \gamma}\right)$ plane (cf. Eqs. (37)-(38)). Upper/lower plots corresponding to $g g / b \bar{b}$ production, while left/right plots to the large/small width scenario. As reference values we assume $M_{S}=750 \mathrm{GeV}$ and $f(r)=30$. The red curve denotes the new physics scale accessible at a futuristic $100 \mathrm{TeV}$ collider, $\Lambda=20 \mathrm{TeV}$, while the three horizontal lines from top to bottom are three reference cross-sections, namely $6,0.6$ and $0.2 \mathrm{fb}$. The yellow triangle on the top-left of each figure is the region in parameter space where $\Gamma_{S} / M_{S}>10 \%$. 